\title{
Transverse patterning in pigeons
}

\author{
P. A. COUVILLON and M. E. BITTERMAN \\ University of Hawaii, Honolulu, Hawaii
}

\begin{abstract}
Pigeons were trained to choose between colored lights (A, B, C, and D), first in a two-pair ambiguouscue problem $(A+B-, B+C-)$, and then, with all colors nondifferentially reinforced, in a three-pair loop problem $(\mathrm{A}+\mathrm{B}-, \mathrm{B}+\mathrm{C}-, \mathrm{C}+\mathrm{A}-)$ followed by a four-pair loop problem $(\mathrm{A}+\mathrm{B}-, \mathrm{B}+\mathrm{D}-, \mathrm{D}+\mathrm{C}-, \mathrm{C}+\mathrm{A}-)$. Systematic efforts were made to simulate the data with a variety of models incorporating one or another of three conceptions of stimulus compounding prominent in the literature on compound conditioning. One conception is that the components of a compound stimulus are altered by interaction; the second is that they are not altered, but only supplemented with a new (compound-unique) component generated in the interaction; and the third is that the components entirely disappear in a configurational transformation. The ambiguous-cue data could be simulated accurately with each of the models, but the loop data with none of them. A convincing explanation of performance in loop problems remains to be found.
\end{abstract}

The parsimonious assumption that a compound conditioned stimulus functions as a sum of its components was called into question early in the history of conditioning. Pavlov (1927) found that dogs could be trained with differential reinforcement to respond to a compound of two stimuli (such as a light and a tone presented together) but not to the separate components (light alone or tone alone), or to respond to the separate components but not to the compound. Compound-component discrimination in dogs was demonstrated by Woodbury (1943) with an instrumental conditioning technique, and thereafter both with classical and instrumental techniques in a variety of animals such as rats, pigeons, and rabbits (Rescorla, 1972; Rescorla, Grau, \& Durlach, 1985; Whitlow \& Wagner, 1972).

The summation rule has been called into question also by the fact that animals can be trained to respond differentially to sets of compounds made up of the same components and differing only in the grouping of the components, a discrimination that Lashley (1938) described as "conditional." In an experiment on eyelid-conditioning in rabbits, for example, Saavedra (1975) used two auditory stimuli ( $A$ and $B$ ) and two visual stimuli (X and $Y$ ); the animals soon came to respond more to the compounds $A X$ and $\mathrm{BY}$, which were reinforced, than to the compounds $\mathrm{AY}$ and BX, which were not. Like results have been obtained in experiments with goldfish (Bitterman, 1984) and pigeons (Rescorla et al., 1985).

In Lashley's (1938) work on conditional discrimination, rats were trained to choose between two compounds pre-

This research was supported in part by Grant IBN-9308132 from the National Science Foundation and in part by Research Centers for Minority Institutions Grant RR03061 from the National Institutes of Health. The authors are indebted to Anne Asam and Radji Tolentino for assistance in data collection, to Bradley $R$. Jones for advice on some difficult computer problems, and to E. James Keboe for helpful suggestions. Correspondence should be addressed to M. E. Bitterman, Békésy Laboratory of Neurobiology, 1993 East-West Road, Honolulu, HI 96822 (e-mail: jeffb@pbrc. hawaii.edu). sented simultaneously: an upright rather than an inverted triangle reinforced when the backgrounds of both were black, and an inverted rather than an upright triangle reinforced when the backgrounds of both were striated. More commonly, as in all of the other work considered thus far, the stimuli to be discriminated are presented sequentially and differential readiness to respond to them is measured. Conditional discrimination in choice situations has been demonstrated also in foraging honeybees fed on targets labeled with colors and odors (Couvillon \& Bitterman, 1988). How the positive and negative compounds are paired in conditional choice experiments has been found to make little difference. Rats trained in Lashley's problem performed well when tested with either of the positive compounds paired with either of the negative compounds (North, Maller, \& Hughes, 1958), and the same was true for the honeybees. The pairing is irrelevant because each of the alternatives embodies all of the elements required to predict the outcome of response to it. Such compounds, which it is convenient to describe as "unitary," may be conditioned in the same way in choice training as in sequential training; only the performance measure is different.

Pavlov (1927) studied conditional discrimination with compounds whose components were themselves presented sequentially rather than simultaneously. A dog could be trained, for example, to salivate in response to a light followed first by a touch and then by a sound, but not to the same three stimuli presented in other sequences. As both Pavlov (1927) and Hull (1943) suggested, serial compounds can be treated as unitary compounds composed of current stimuli and the "traces" (rapidly decaying afferent residuals) of previous stimuli. Not so easily decided, however, is how to deal with a class of conditional choice problems in which the relevant components are spatially rather than temporally disaggregated-problems that require what Spence (1952) called "transverse patterning." Whether or not choice of a given alternative is reinforced in a transverse problem is contingent on the alternative 
that is paired with it. Spence proposed, for example, that rats trained with the three possible pairings of a circle, a square, and a triangle might learn to choose the circle in preference to the square, the square in preference to the triangle, and the triangle in preference to the circle. Successful discrimination in a "loop" problem of this sort was reported soon thereafter in work with chimpanzees (Thompson, 1953) and again more recently in a rather unusual experiment with rats (Alvarado \& Rudy, 1992). Fersen, Wynne, Delius, and Staddon (1991), however, failed to find significantly better-than-chance performance in a six-pair loop problem with pigeons. Other examples of transverse patterning are provided by successful performance in a variety of quasi-conceptual problems, such as the discrimination of oddity (e.g., choose a circle presented with two rectangles, but a rectangle presented with two circles) by monkeys and rats (Klüver, 1933; Wodinsky \& Bitterman, 1953). Our interest here is in the formal analysis of transverse patterning from the perspective afforded by efforts to understand unitary compounding.

Three different explanations of unitary compounding have been proposed, and fundamental to each of them is the Pavlovian concept of afferent interaction. The first explanation, carefully developed by Hull (1943), is that the components of a compound stimulus (literally, the afferent processes to which they give rise when separately presented) are altered by interaction but continue to operate as components. Stimuli $A$ and $B$ become $A^{\prime}$ and $B^{\prime}$ when presented together, and the associative strength of the compound is given by the summed strengths of $A^{\prime}$ and $B^{\prime}$. (The summation rule is retained.) Associative strength generalizes between the altered and unaltered processes as a function of their similarity, which is itself an inverse function of the degree of interaction.

The second explanation (Rescorla, 1972; Whitlow \& Wagner, 1972) is that the integrity of the primary afferent components is entirely preserved (A and B are unchanged), but the interaction generates a new, compoundunique component, $\mathrm{Q}$, which gains and loses associative strength with reinforcement and nonreinforcement of the compound in the same way as do the primary components. The associative strength of the compound is given by the summed strengths of $A, B$, and $Q$. (The summation rule is retained.) Rescorla and Wagner (1972) assumed that all components of a compound stimulus share any changes in associative strength produced by reinforcement or nonreinforcement of the compound, although the concept of compound-unique components has proved useful in analyzing the results of experiments on compound conditioning in honeybees even on the "independence" assumption of traditional continuity theory - the assumption that the components of a compound gain or lose associative strength independently with reinforcement or nonreinforcement of the compound (Couvillon \& Bitterman, 1988, 1991).

The third explanation, a heritage of Gestalt psychology to which sympathetic consideration has continued to be given (see, e.g., Bellingham, Gillette-Bellingham, \& Kehoe, 1985; Pearce \& Wilson, 1990; Wilson \& Pearce,
1989), is that the associative strength of the compound as a whole-as a "configuration"--changes with reinforcement and nonreinforcement, the components being lost entirely in the interaction; $A$ and $B$ together are treated neither as $A^{\prime}+B^{\prime}$, nor as $A+B+Q$, but as $Q$ alone. (The summation rule is abandoned.) The associative strengths of the separate components (which are treated as other "configurations") change with reinforcement and nonreinforcement of the compound only as a function of their similarity to the compound, and by the same token the effects of experience with the components generalize to the compound.

All three explanations have a place for pair-wise interaction and so provide some basis for understanding transverse patterning. In a loop problem, a circle (literally, its afferent correlate) may be different in the presence of a square than in the presence of a triangle (the Hullian assumption); or it may be enriched by a pair-unique component that is different in the presence of a square than in the presence of a triangle; or, to borrow an idea from Gulliksen and Wolfle (1938), each of the three pairs of figures (circle-square, square-triangle, and triangle-circle) may function as a separate configuration. The first two interpretations have in common the assumption that the animal chooses between unitary compounds, but the third is different and requires some elaboration.

Gulliksen and Wolfle (1938) assumed that the two lateral arrangements of a single pair of stimuli in a simple simultaneous problem (e.g., $\mathrm{A}+\mathrm{B}-$ and $\mathrm{B}-\mathrm{A}+$ ) function as separate configurations to which the animal responds by going to one or the other of the two positions (left or right) occupied by the stimuli (left to $A+B-$ and right to $A-B+$ ). Although that assumption was subsequently shown by Nissen (1950) to be incorrect, there is in fact some persuasive evidence for the discrimination of the properties of pairs of stimuli as pairs (Bitterman, 1953), of which the most striking is perhaps provided by performance of rats in "two-situational" problems (Teas \& Bitterman, 1952; Turbeville, Calvin, \& Bitterman, 1952; Zeiler \& Paul, 1965). Two-situational training may be thought of as training in two concurrent simultaneous discriminations with only one of the two possible lateral arrangements of each pair of stimuli presented and with position nondifferentially reinforced. On some trials, for example, the animal chooses between light gray (L) and dark gray (D) cards placed side by side, with $L$ always at left and always reinforced $(\mathrm{L}+\mathrm{D}-)$; on the remaining trials, the choice is between wide (W) and narrow (N) vertical black-and-white stripes, with $\mathrm{N}$ always at right and always reinforced $(\mathrm{W}-\mathrm{N}+$ ). Such a problem can be mastered on the basis of the components alone ( $\mathrm{L}$ and $\mathrm{N}$ reinforced, D and $\mathrm{W}$ nonreinforced, and position nondifferentially reinforced), in which case it should be equivalent to the corresponding four-situational problem $(\mathrm{L}+\mathrm{D}-, \mathrm{D}-\mathrm{L}+, \mathrm{W}-\mathrm{N}+, \mathrm{N}+\mathrm{W}-)$, but it is not. For one thing, the two-situational problem is very much easier. Furthermore, on early encounters with DL and NW after training with $\mathrm{L}+\mathrm{D}-$ and $\mathrm{W}-\mathrm{N}+$, the animals tend to choose $\mathrm{D}$ and $\mathrm{W}$, both previously nonreinforced - that is, 
they behave as though they have learned to go left to a pair of gray cards (in either lateral arrangement) and to go right to a pair of striped cards.

Other evidence shows, however, that the control by pair properties in these problems is not exclusive. For example, in four-situational training that follows two-situational training, performance is better when the positive and negative components remain the same than when they are reversed (Turbeville et al., 1952). These results suggest that components and configurational properties can be perceived and learned about at the same time. It seems reasonable, therefore, to propose that what the animals learn in the loop problem is to choose the circle when circle and square are paired in either lateral arrangement $(\mathrm{A}+\mathrm{B}-$ or $\mathrm{A}-\mathrm{B}+$ ), the square when the square-triangle pair is presented, and so forth. This analysis departs from a strict configurational interpretation in that performance is assumed to be controlled, not only by the perception of each pair of stimuli as a pair, but also by perceptions of the separate stimuli, which (rather than positions) serve to direct response.

Our purpose in this paper is first of all to provide a substantial and readily expandable set of data on the performance of pigeons in loop problems. The second purpose is to describe the results of our efforts to simulate the data with an array of theories incorporating one or the other of the three general interpretations of transverse patterning that have been considered. Our work profits from earlier quantitative analyses of the performance of rats, rabbits, and pigeons in compound-component discriminations and related problems (e.g., Bellingham et al., 1985; Pearce \& Redhead, 1993; Wilson \& Pearce, 1989), but goes beyond them in an important respect: A performance rule is sought that will permit exact rather than merely ordinal predictions of experimental outcomes (cf. Couvillon \& Bitterman, 1991, 1992; Zhuikov, Couvillon, \& Bitterman, 1994).

\section{EXPERIMENTAL METHOD}

\section{Subjects}

The subjects were 9 homing pigeons obtained from a local breeder. They were maintained at $80 \%$ of free-feeding weight on a $24-\mathrm{h}$ feeding schedule. Before being put to the present use, they were trained in a standard keypecking situation with tones and variations in the ambient magnetic field as discriminative stimuli.

\footnotetext{
Apparatus

In the present experiment, the animals were trained in a chamber, $28 \mathrm{~cm}$ long $\times 28 \mathrm{~cm}$ wide $\times 30 \mathrm{~cm}$ high, that was set in a soundattenuating enclosure and dimly illuminated by a recessed white houselight. Three walls of the chamber were blank, and the fourth displayed only the apertures of two conventional grain feeders. The apertures, each $6 \mathrm{~cm}$ wide $\times 5 \mathrm{~cm}$ high, were $5 \mathrm{~cm}$ apart and $5 \mathrm{~cm}$ above the floor. Each aperture could be illuminated independently with one of eight different colors produced by white light transmitted through appropriate Kodak Wratten filters. The illuminated apertures were themselves the discriminative stimuli. The response of the subject was to insert its head into one or the other of the apertures, and the choice was registered by an infrared photodetector,
}

which also elevated the grain tray if the choice was correct. The technique was designed to maximize cue-response-reward contiguity, which has been shown repeatedly since the turn of the century to facilitate discriminative learning in a wide variety of animals (Gonzalez, Berger, \& Bitterman, 1966; Stearns \& Bitterman, 1965). All events of the experiment were programmed by a desktop computer that also stored the data.

\section{Procedure}

After pretraining with the colors to be used in the experiment (which were presented singly, half the time in each aperture, with all responses reinforced), the experimental training began.

In Stage 1, the animals were trained with three colors $(A, B$, and $C)$ in an ambiguous-cue problem, $\mathrm{A}+\mathrm{B}-$ on half the trials and $\mathrm{B}+\mathrm{C}-$ on the rest. The actual colors used were red, blue, and green, and they were balanced over animals in Latin-square fashion (red was A for 3 subjects, green was A for 3 others, blue was A for the rest, and so forth). In each of 44 daily sessions, there were 40 trials, 20 with $\mathrm{A}+\mathrm{B}-$ and 20 with $\mathrm{B}+\mathrm{C}-$ in balanced quasi-random sequences. Position also was balanced, with each pair occurring 10 times in each of the two lateral arrangements. The intertrial interval was $30 \mathrm{sec}$. Each trial began with the illumination of the two feeder apertures. Insertion of the animal's head into the correct aperture ( $\mathrm{A}$ on $\mathrm{A}+\mathrm{B}-$ trials or $\mathrm{B}$ on $\mathrm{B}+\mathrm{C}-$ trials) was rewarded with $3.5 \mathrm{sec}$ of access to mixed grain, during which time the apertures remained illuminated. If an incorrect choice was made, both apertures were darkened for $6 \mathrm{sec}$, after which the correct aperture alone was illuminated and response to it was reinforced (guidance procedure). The use of guidance in difficult choice problem counteracts the tendency of the animals to fall into position habits.

In Stage 2, the loop was closed by the addition of $\mathrm{C}+\mathrm{A}-$ trials. In each of 40 sessions, there were 42 trials, 14 with each of the three pairs $(\mathrm{A}+\mathrm{B}-, \mathrm{B}+\mathrm{C}-$, and $\mathrm{C}+\mathrm{A}-)$ in balanced quasi-random sequences and position balanced as well. In all other respects, the procedure was the same as that in Stage 1.

In Stage 3, a new stimulus, D (yellow) was added; color was not balanced in this stage, because we did not anticipate this extension of the training at the outset of the experiment. With the new stimulus, the loop was expanded to four pairs $-A+B-, B+D-, D+C-$, and $\mathrm{C}+\mathrm{A}-$. Now there were two old pairs differentially reinforced as before $(A+B-$ and $C+A-)$, and two new pairs $(B+D-$ and $D+C-)$, again with each of the four stimuli nondifferentially reinforced. In each of 36 sessions, there were 40 trials, 10 with each of the four pairs in balanced quasi-random sequences and position balanced as well. In all other respects, the procedure was the same as it was in the two preceding stages of training.

\section{EXPERIMENTAL RESULTS}

In Figure 1, performance in Stage 1 is plotted in terms of the mean probability of correct choice for each of the two pairs of colors $(\mathrm{A}+\mathrm{B}-$ and $\mathrm{B}+\mathrm{C}-$ ) in each training session. An analysis of variance (ANOVA) shows a significant pairs effect $[F(1,8)=7.20, p=.0278]$, a significant sessions effect $[F(43,344)=11.80, p<.0001]$, and a significant pairs $\times$ sessions interaction $[F(43,344)=$ $3.46, p<.0001]$. Better $\mathrm{B}+\mathrm{C}-$ than $\mathrm{A}+\mathrm{B}-$ performance has been found before in pigeons and other animals trained with three distinctive stimuli (see, e.g., Boyer \& Polidora, 1972; Fischer, Couvillon, \& Bitterman, 1993; Hall, 1980 ) and may reflect nothing more than control by the differentially reinforced colors apart from any interaction. The atypical convergence of the two curves suggests a 


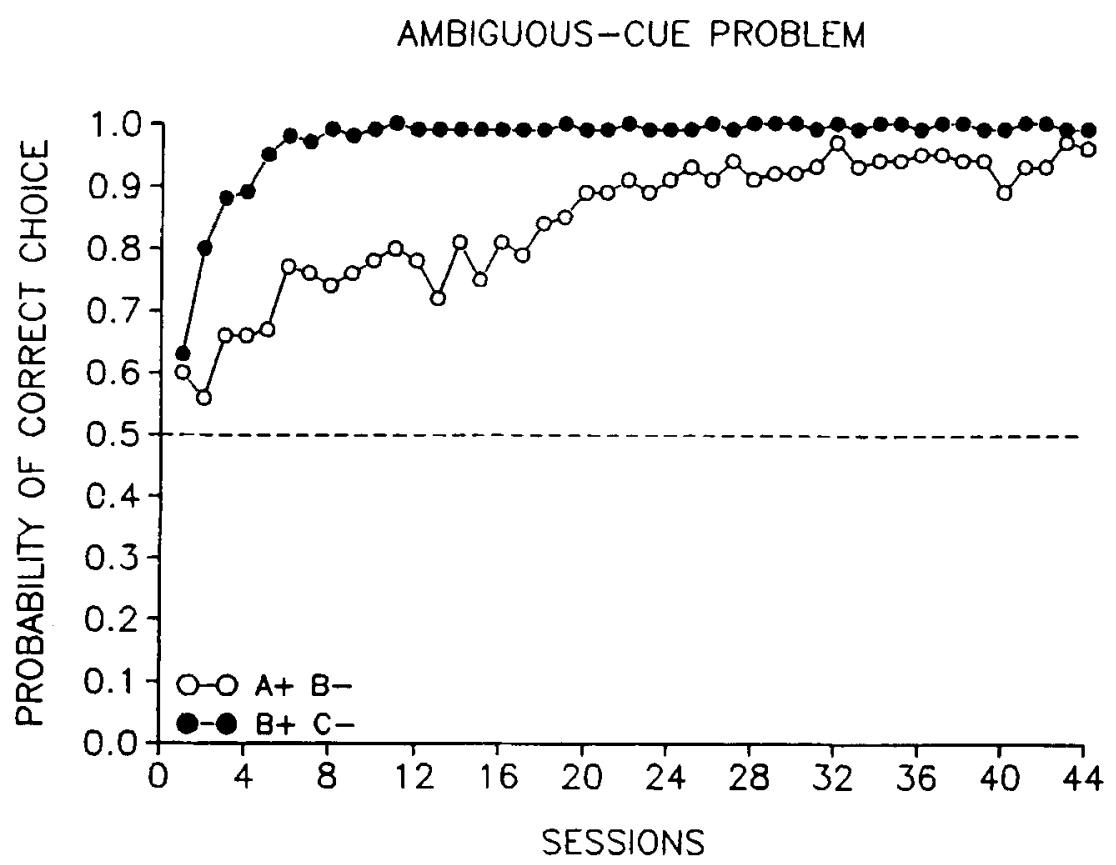

Figure 1. The mean probability of correct choice for each of the two pairs of colors $(A+B-$ and $B+C-)$ in each training session of Stage 1 .

patterning effect, but the possibility that the data can be understood in terms of control only by components should not be dismissed on intuitive grounds alone.

In Figure 2, performance in Stage 2 is plotted in terms of the mean probability of correct choice for each of the three pairs of colors (the two old pairs, $\mathrm{A}+\mathrm{B}-, \mathrm{B}+\mathrm{C}-$, and the new pair, $\mathrm{C}+\mathrm{A}-$ ) in each training session. An ANOVA shows a significant pairs effect $[F(2,16)=3.99, p=$ $.0393]$, a significant sessions effect $[F(39,312)=11.87$, $p<.0001]$, and a significant pairs $\times$ sessions interaction $[F(78,624)=5.03, p<.0001]$. There was no significant difference between the two old pairs $[F(1,8)=2.05, p=$ $.1905]$. Performance in the first sessions after the introduction of the new pair - the decrement in $A+B-$ and $\mathrm{B}+\mathrm{C}-$ performance, and the poorer-than-chance $\mathrm{C}+\mathrm{A}-$ performance-suggests control by components, but the subsequent convergence of the three curves at a high terminal level affords a prime example of patterning.

In Figure 3, performance in Stage 3 is plotted in terms of the mean probability of correct choice in each training session for each of the two previously encountered pairs of colors $(\mathrm{A}+\mathrm{B}-$ and $\mathrm{C}+\mathrm{A}-)$ and each of the two new pairs $(B+D-$ and $D+C-)$. The curves are based on the data for only 8 subjects, because one was lost in this stage of training. A $2 \times 2$ ANOVA shows a significant old-new difference $[F(1,7)=24.87, p=.0016]$, a significant sessions effect $[F(35,245)=10.32, p<.0001]$, and a significant old-new $\times$ sessions interaction $[F(35,245)=$ $1.80, p=.0055]$. The two old pairs did not differ significantly from each other $(F<1)$, and neither did the two new pairs $(F<1)$. It remains for systematic analysis to determine just what role the components may have contin- ued to play in this stage of training, but again the patterning is unmistakable.

\section{THEORETICAL ANALYSIS}

We consider now how well each of several different theories can deal with the data of these experiments. We express each theory to be evaluated in equational form and then search systematically for sets of parameter values yielding accurate simulations of the data, which it should be possible to find if the theory is correct. Despite the common impression that good quantitative fits are easy to obtain, they are not, as will soon become evident; the misconception reflects a failure to distinguish between theory-based simulation and unconstrained curve-fitting. Our strategy in the case of each theory was to look for the best fits to the data of the first two stages of training as measured by the root-mean-square (RMS) deviation of simulated from actual performance, and then with the same parameter values to predict performance in the third stage.

It may be well to emphasize that the theories under consideration were chosen simply to inquire into the usefulness of the various current interpretations of unitary compounding for the understanding of transverse patterning. Although none of them could possibly be offered as a general theory of learning, it does not follow from their obvious limitations that none could be expected to fit our data or in some other way to serve that purpose. The PUC model, soon to be described, clearly fails to deal with overshadowing, blocking, or conditioned inhibition, but it yields an accurate fit to the data of Fersen et al. (1991) on 


\section{THREE-PAIR LOOP PROBLEM}

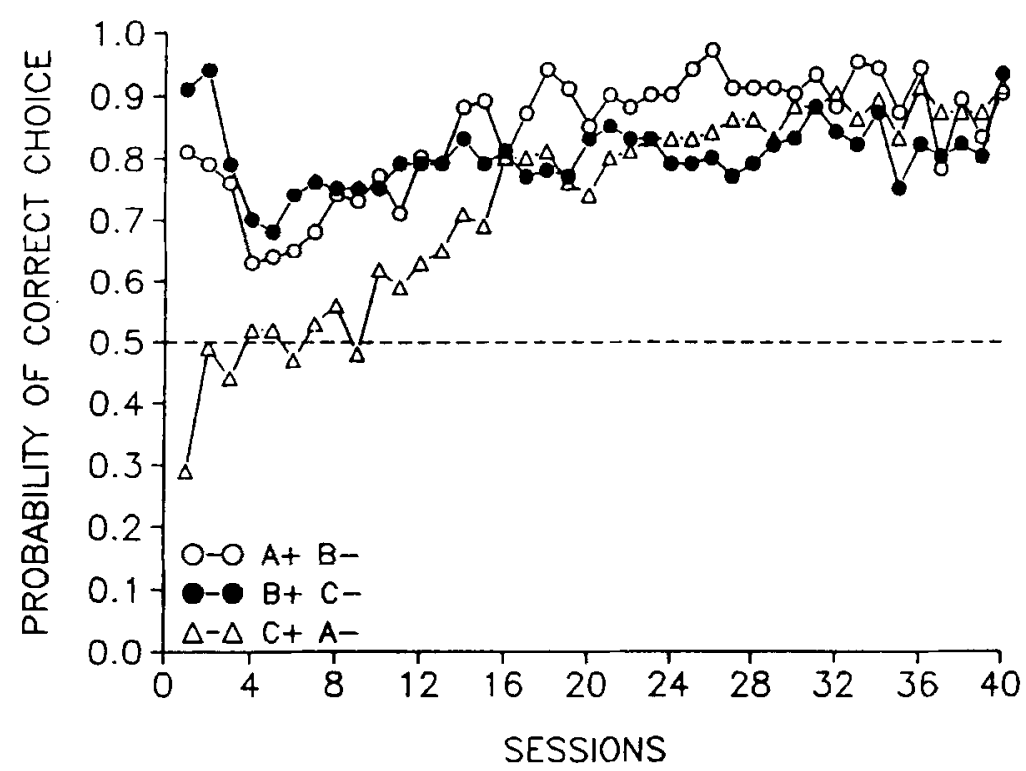

Figure 2. The mean probability of correct choice for each of the three pairs of colors (the two old pairs, $\mathrm{A}+\mathrm{B}-, \mathrm{B}+\mathrm{C}-$, and the new pair, $\mathrm{C}+\mathrm{A}-$ ) in each training session of Stage 2.

transitivity in pigeons (Couvillon \& Bitterman, 1992), suggesting that the phenomenon is much simpler than earlier suspected. There is no basis for deciding in advance whether our data on transverse patterning reflect the operation of mechanisms regarding which the PUC model or any of the others is silent.

Common to all of the theories under consideration is the assumption that the attractiveness of a stimulus is given by the strength $(V)$ of its association with reward. Change in $V$ contingent on reinforcement or nonreinforcement is described by the linear equation of Bush and Mosteller (1951)

$$
\Delta V_{\mathrm{A}}=\alpha_{\mathrm{A}} \cdot \beta\left(\lambda-V_{\mathrm{A}}\right)
$$

in the now-more-familiar notation of Rescorla and Wagner (1972): $V_{\mathrm{A}}$ is the associative strength of Stimulus $A$ at the start of a given trial; $\Delta V_{\mathrm{A}}$ is the change in $V_{\mathrm{A}} ; \lambda$ (scaled from $0-1$ ) is the asymptotic associative strength, which varies with the magnitude of reinforcement and is taken as 0 when there is no reinforcement; $\beta$ (scaled from $0-1$ ) is the learning-rate parameter, which may be different for reinforcement than for nonreinforcement- the incremental rate is termed $U \beta$ and the decremental rate $D \beta$; and $\alpha_{\mathrm{A}}$ (scaled from $0-1$ ) is the salience of $\mathrm{A}$, which also affects the rate of acquisition. If, on a simulated $\mathrm{A}+\mathrm{B}-$ trial, the animal chooses $\mathrm{A}$, then $\Delta V_{\mathrm{A}}=\alpha_{\mathrm{A}}$. $U \beta\left(\lambda-V_{\mathrm{A}}\right)$; if it chooses $\mathrm{B}, \Delta V_{\mathrm{B}}=\alpha_{\mathrm{B}} \cdot D \beta\left(0-V_{\mathrm{B}}\right)=$ $-\alpha_{\mathrm{B}} \cdot D \beta \cdot V_{\mathrm{B}}$, and, because incorrect choice is followed by a guided trial with $\mathrm{A}, \Delta V_{\mathrm{A}}=\alpha_{\mathrm{A}} \cdot U \beta\left(\lambda-V_{\mathrm{A}}\right)$.

Choice between two targets - say, $A$ and $B-$ is predicted on the basis of their relative associative strength, $r$. The relative strength of $\mathrm{A}, r_{\mathrm{A}}$, is computed as $V_{\mathrm{A}} /\left(V_{\mathrm{A}}+V_{\mathrm{B}}\right)$, and the relative strength of $\mathrm{B}, r_{\mathrm{B}}$, as $V_{\mathrm{B}} /\left(V_{\mathrm{A}}+V_{\mathrm{B}}\right)$, or $1-r_{\mathrm{A}}$. $P_{\mathrm{A}}$, the probability of choosing $\mathrm{A}$, is a power function of $r_{\mathrm{A}}$ designated by two parameters, $K$ and $s$. Plotted in Figure 4 are samples of such functions, differing in slope and curvature. They range from $K=.5, s=1.3$, close to $K=0, s=.5$ - which it is convenient to think of as "maximizing" $\left(P_{\mathrm{A}}=1\right.$ whenever $\left.r_{\mathrm{A}}>.5\right)$-through a set of intermediate functions, to $K=1, s=.5$ - which may be thought of as "matching" $\left(P_{\mathrm{A}}=r_{\mathrm{A}}\right)$. All of the functions are generated with the scaling equations

$$
P=.5+s(2 r-1)^{K}
$$

for $r \geq .5$ and

$$
P=.5-s(1-2 r)^{K}
$$

for $r \leq .5$, and in Figure 4 they are plotted only for $r \geq .5$ because they are perfectly symmetrical for $r<.5$. The truncation of $P$ with $s>.5$ implies, of course, a performance ceiling - insensitivity of the choice measure to differences among higher values of $r$. Although little attention has been given in the modeling literature to possibilities other than matching, it may be well to note that the best fit to a large set of honeybee data is yielded by an intermediate function much like $K=.75, s=.625$ (Couvillon \& Bitterman, 1991), which has also provided a good fit to the data of Fersen et al. (1991) on transitivity in pigeons (Couvillon \& Bitterman, 1992). It may be well also to emphasize the difference between our (theoretical) choice functions and the plots of choice ratios against reinforcement ratios found in the probability-learning literature.

Our computational method of simulation (Couvillon \& Bitterman, 1989) is preferable to the familiar Monte Carlo 
FOUR-PAIR LOOP PROBLEM

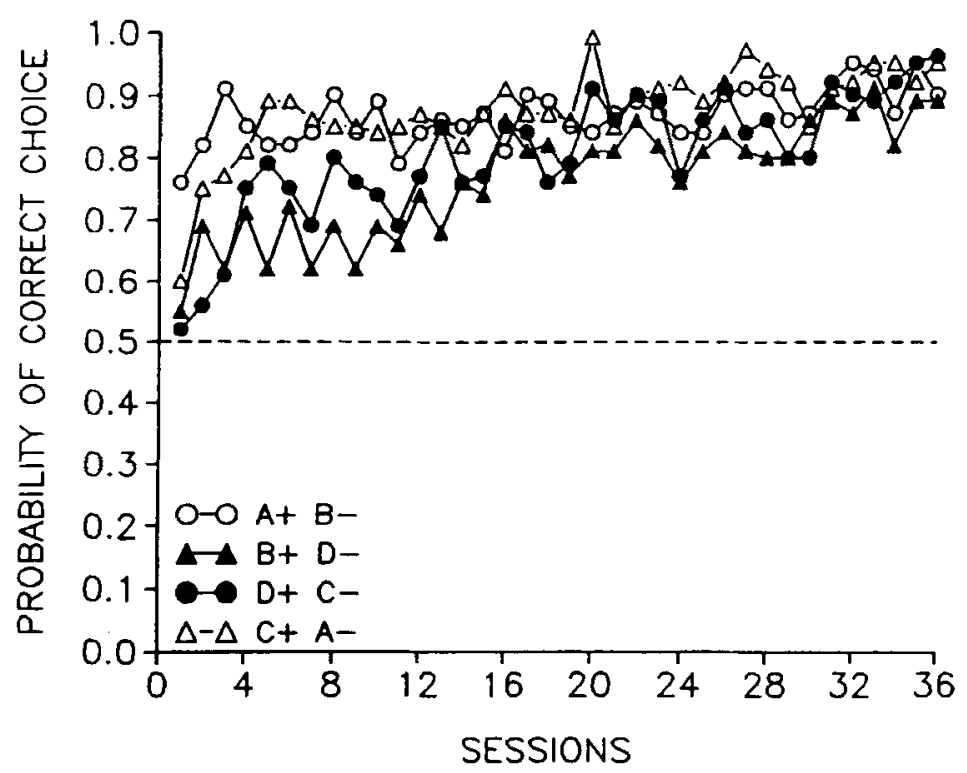

Figure 3. The mean probability of correct choice for each of the two previously encountered pairs of colors $(A+B-$ and $C+A-)$ and each of the two new pairs $(B+D-$ and $D+C-)$ in each training session of Stage 3 .

method both because it is much faster and because it is free of the random error of simulation inherent in the Monte Carlo method. The Monte Carlo method is to deal with each of a set of "stat-animals" in turn, using a randomnumber generator to determine which choice is actually made on each trial and then changing the associative strengths of one or both of the alternatives depending on which choice is made (incrementing $V_{\mathrm{A}}$ alone if $\mathrm{A}$ is chosen, or, if $\mathrm{B}$ is chosen, decrementing $V_{\mathrm{B}}$ and then incrementing $V_{\mathrm{A}}$ to reflect the guidance that would follow). Then $r$ is calculated for the next trial, the random-number generator is consulted again, and so forth. The simulated performance is the mean performance of a large number of stat-animals - the larger the number, the smaller the random error of simulation. Instead we simulate the mean performance directly by calculating mean associative strengths on the assumption that the proportion of animals choosing $\mathrm{A}$ on each trial is $P_{\mathrm{A}}$. On an $\mathrm{A}+\mathrm{B}-$ trial, for example, $\Delta V_{\mathrm{B}}=\alpha_{\mathrm{B}} \cdot\left(1-P_{\mathrm{A}}\right) \cdot D \beta\left(0-V_{\mathrm{B}}\right)$ and, given the guidance procedure, $\Delta V_{\mathrm{A}}=\alpha_{\mathrm{A}} \cdot U \beta\left(\lambda-V_{\mathrm{A}}\right)$. The logic is the same for all of the models evaluated.

Hullian generalization (HG model). The Hullian conception is that we are dealing in the loop problem with what are in effect nine stimuli-the three colors as presented separately $(\mathrm{A}, \mathrm{B}$, and $\mathrm{C})$ and as modified by interaction in the pairs $\left(A^{\prime} B^{\prime}, B^{\prime \prime} C^{\prime}\right.$, and $\left.C^{\prime \prime} A^{\prime \prime}\right)$. With a conventional treatment of generalization in terms of identical elements,

$$
\mathrm{A}^{\prime}=g \mathrm{~A}+(1-g) \mathrm{Q} \text {, }
$$

which is to say that a proportion $(g)$ of the elements of $A^{\prime}$ are shared with $A$ and the rest $(Q)$ are unique. It follows that

$$
V_{\mathrm{A}^{\prime}}=g V_{\mathrm{A}}+(1-g) V_{\mathrm{Q}},
$$

with $g$ as the generalization constant. By the same token,

$$
\mathrm{B}^{\prime}=g \mathrm{~B}+(1-g) \mathrm{R},
$$

and

$$
V_{\mathrm{B}^{\prime}}=g V_{\mathrm{B}}+(1-g) V_{\mathrm{R}},
$$

where $R$ represents the set of elements unique to $B^{\prime}$. Comparable equations can be written for $B^{\prime \prime}, C^{\prime}, C^{\prime \prime}$, and $A^{\prime \prime}$, and with $\mathrm{S}, \mathrm{T}, \mathrm{U}$, and $\mathrm{W}$, respectively, representing their unique elements. $(R, T$, and $W$, which never acquire any associative strength, are specified only for formal purposes.) The pretraining of the animals (which simply incremented $V_{\mathrm{A}}, V_{\mathrm{B}}$, and $\mathrm{V}_{\mathrm{C}}$ ) and their subsequent training in the first and second stages of the experiment were simulated with $\alpha=1, \lambda=1$, and factorial variation in the three parameters, $U \beta, D \beta$, and $g$ (the learning rates from $.0002-.24, g$ from $.05-1$ ), for each of the choice functions plotted in Figure $4-77,440$ simulations in all.

Pair-unique components (PUC model). In the PUC model, the simplest of several models incorporating the assumption of pair-unique components, the animals are treated as choosing between the compounds $A Q$ and $B R$ on $A+B-$ trials - $A$ is enriched by the presence of $B$, and $B$ by the presence of $A$ - between BS and CT on B+Ctrials, and between $\mathrm{CU}$ and $\mathrm{AW}$ on $\mathrm{C}+\mathrm{A}-$ trials, with $\mathrm{Q}$, $\mathrm{R}, \mathrm{S}, \mathrm{T}, \mathrm{U}$, and $\mathrm{W}$ designating pair-unique components. The components are assumed to gain $(U \beta)$ or lose $(D \beta)$ associative strength independently with reinforcement or nonreinforcement (response to $\mathrm{A}$ on $\mathrm{A}+\mathrm{B}-$ trials increases the strengths of $A$ and $Q$ ), and the strength of 


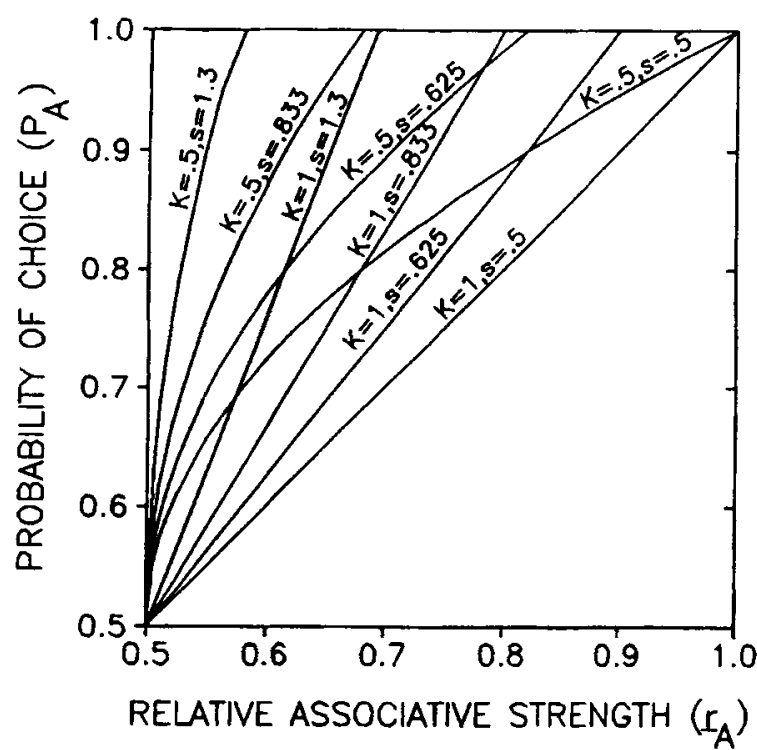

Figure 4. Performance curves, each showing the hypothetical probability of choosing one of two alternatives $\left(P_{A}\right)$ as a function of its relative associative strength $\left(r_{\mathrm{A}}\right)$.

each compound is taken as the sum of the strengths of its components (e.g., $\left.V_{\mathrm{AQ}}=V_{\mathrm{A}}+\mathrm{V}_{\mathrm{Q}}\right)$. $(\mathrm{R}, \mathrm{T}$, and $\mathrm{W}$, which never acquire any associative strength, are specified only for formal purposes.) In the simulations (with $\lambda=1$ ), $\alpha_{U}$, the salience of the pair-unique components, and $\alpha_{p}$, the salience of the primary components (the colors), were varied factorially (range, .05-1) along with the two learning rates (four parameters in all) for each of the choice functions plotted in Figure 4-1,548,800 simulations in all. There is obvious similarity between the HG and PUC models, but they yield different predictions.

Pair-unique components with shared associative strength (RW model). The RW model is the same as the PUC model $(\mathrm{AQ}+\mathrm{BR}-, \mathrm{BS}+\mathrm{CT}-, \mathrm{CU}+\mathrm{AW}-)$ except that the independence assumption is abandoned in favor of the Rescorla-Wagner assumption of shared associative strength. For example, the discrepancy between $\lambda$ and $V_{\mathrm{AQ}}-V_{\mathrm{A}}+V_{\mathrm{Q}}$-is used in computing $\Delta V_{\mathrm{A}}$ and $\Delta \mathrm{V}_{\mathrm{Q}}$ when there is a reinforced response to $A Q$, and the discrepancy between zero and $V_{\mathrm{BR}}$ in computing $\Delta V_{\mathrm{B}}$ and $\Delta V_{\mathrm{R}}$ when there is a nonreinforced response to $\mathrm{BR}$. In consequence, $\mathrm{R}, \mathrm{T}$, and $\mathrm{W}$ now play a significant role, tending to acquire negative associative strength. Furthermore, $r$ is no longer bounded by zero and 1 , and values outside that range are uninterpretable; to solve this problem, Wynne (1995) has used a transformation of $r$ that is unsatisfactory because it leads in some cases to the prediction that an inhibitory alternative will be chosen with high probability over an excitatory one. As it happened, however, our simulations generated no values of $r$ outside the meaningful range, with $V_{\mathrm{BR}}, V_{\mathrm{CT}}$, and $V_{\mathrm{AW}}$ all remaining positive. As in the PUC simulations, there was wide factorial variation of $U \beta, D \beta, \alpha_{U}$, and $\alpha_{P}$ (four pa- rameters in all) for each of the choice functions plotted in Figure $4-1,548,800$ simulations in all.

The simulations were then repeated on the perhaps more realistic assumption (cf. Wynne, 1995) of a fourth primary component, $\mathrm{X}$, common to each of the six compounds (RW-X model). Many sets of parameters yielded uninterpretable values of $r$ (outside the range of $0-1$ ), but for interpretable values of $r$ (in the $0-1$ range) the change made little difference. The best-fitting curves turned out to be very much the same as those obtained without the common component, as did the sets of parameter values that generated them.

Pair-unique components with learned salience (LS model). The LS model is like the PUC model except that the saliences of the primary components, $\alpha_{P}$, and the pair-unique components, $\alpha_{U}$, increase and decrease independently (cf. Heinemann \& Chase, 1970) with reinforcement and nonreinforcement. The equations for change in $\alpha$ are the same as for change in $V$, except that the learning rates may be different. In the simulations, which began with $\alpha_{P}=1$ and $\alpha_{U}=0$, the four learning rates ( $U \beta$ and $D \beta$ for $V$ and for $\alpha$ ) were varied factorially for each of the choice functions plotted in Figure 4$1,874,048$ simulations in all.

Pair-unique components with selective attention (SM model). The SM model is like the PUC model except that the primary and the pair-unique components $(\alpha=1)$ compete for attention (Sutherland \& Mackintosh, 1971). On a given trial, say, with $A Q+B R-$, the animal attends to the primary components $(A$ and $B$ ) with the probability $a_{P}$, or to the pair-unique components $(\mathrm{Q}$ and R) with the complementary probability, $a_{U}: a_{P}=$ $1-a_{U}$. If the animal attends to the primary components, its performance is determined only by their relative associative strength; with correct response, $V_{\mathrm{A}}$ is incremented and so also, in the same way, is $a_{P}$; with incorrect response, $V_{\mathrm{B}}$ and $a_{P}$ are decremented, after which guidance is simulated by incrementing $V_{\mathrm{A}}$ and $a_{p}$. If, at the outset of the trial, the animal attends to the pairunique components, the response is necessarily correct, because $\mathrm{R}$ never acquires any associative strength; accordingly, both $V_{Q}$ and $a_{U}$ are incremented. In the simulations, which began with $a_{P}=1$ and $a_{U}=0$, four learning rates $(U \beta$ and $D \beta$ for $V$ and for $a)$ were varied factorially for each of the choice functions plotted in Figure 4-1,874,048 simulations in all.

Pairs as configurations (GW model). Here we adopt the notion of Gulliksen and Wolfle (1938) that the animals discriminate the properties of pairs of stimuli as pairs. Each pair of colors (e.g., $\mathrm{A}+\mathrm{B}-$ ) is assumed to generate a configuration (e.g., $Q$ ) that elicits one of two alternative responses defined as choosing $A(Q \rightarrow A)$ or choosing $B(Q \rightarrow B)$. The pair $B+C-$ generates $R$, with $R \rightarrow B$ and $\mathrm{R} \rightarrow \mathrm{C}$ as alternative responses, and the pair $\mathrm{C}+\mathrm{A}-$ generates $\mathrm{S}$, with $\mathrm{S} \rightarrow \mathrm{C}$ and $\mathrm{S} \rightarrow \mathrm{A}$ as alternative responses. The six associations (conceived as stimulus-response rather than stimulus-reinforcer associations) are strengthened or weakened with reinforcement or nonreinforcement 
in the usual fashion. Given that the colors are readily distinguishable, the difficulty of the loop problem is traced in this analysis to the difficulty of distinguishing the configural properties. The effect of experience with any of the configurations is assumed to generalize to each of the others as a function of similarity, which is expressed in terms of the proportion of common elements $(g)$ exactly as in the HG model. It may be well to emphasize that the reference here is to neural elements (the proportion of which cannot be specified a priori) and not to stimulus elements as conceived by Pearce and his associates (e.g., Pearce $\&$ Wilson, 1990), whose treatment of similarity is more reminiscent of Wundtian than of Gestalt psychology. For them-if they were indeed to consider the GW model a reasonable extension of their thinking about unitary compounding to choice problems - the $g$ for each pair of colors would be taken at once as .5 because the colored stimuli constituting the three pairs are of equal area. In our simulations, with $\alpha=1$ and $\lambda=1, g$ was varied factorially along with $U \beta$ and $D \beta$ for each of the choice functions of Figure 4-77,440 simulations in all.

The simulations then were repeated with the additional assumption that the component colors themselves inde- pendently gain or lose attractiveness with reinforcement or nonreinforcement (GW-C model). When the animal chooses $\mathrm{A}$ on an $\mathrm{A}+\mathrm{B}-$ trial, both $V_{\mathrm{A} \rightarrow \mathrm{A}}$ and $V_{\mathrm{Q} \rightarrow \mathrm{A}}$ are incremented; when it chooses $\mathrm{B}$, both $V_{\mathrm{B} \rightarrow \mathrm{B}}$ and $V_{\mathrm{Q} \rightarrow \mathrm{B}}$ are decremented. The change made little difference. The best-fitting curves were much the same in the two cases, as were the sets of parameter values that generated them.

In the simulations, the results for the GW model itself were, not merely similar, but identical to those for the HG model. Whatever the differences between the two interpretations may seem to be at the verbal level, in their implementation they are exactly the same. An advantage of the simulation approach, if nothing else, is that it strips away surplus meaning. In any case, we are left now to consider the results for five main models: $\mathrm{HG}(\mathrm{GW})$, PUC, RW, LS, and SM.

\section{SIMULATION RESULTS}

Each of the five models yielded a cluster of good fits to the ambiguous-cue data alone. The mean of the best 10 fits (defined in terms of minimal RMS deviation) for the $\mathrm{HG}(\mathrm{GW})$ model is plotted, along with the experimen-

HG(GW) MODEL

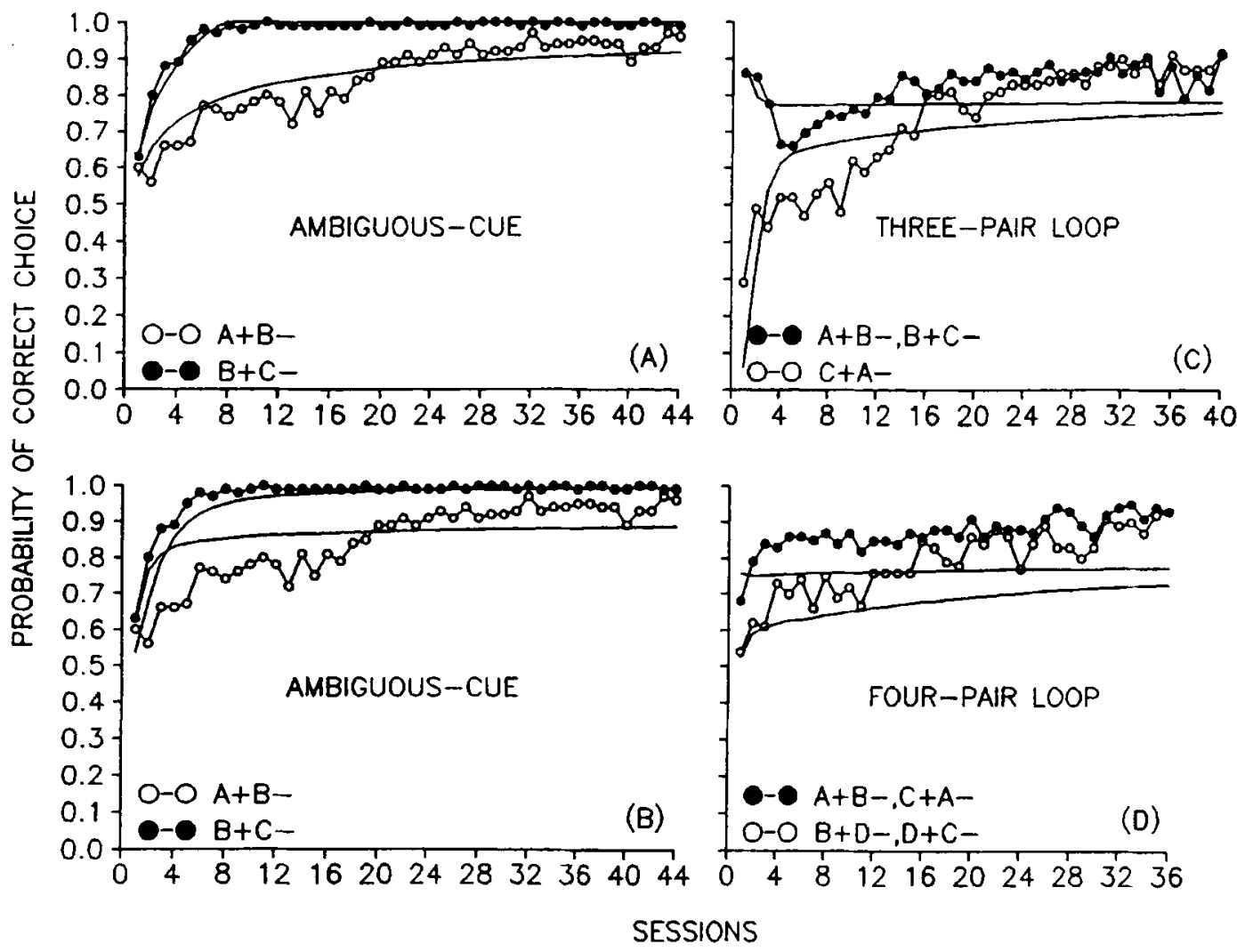

Figure 5. Simulations with the HG(GW) model (smooth curves). (A) Ambiguous-cue performance with parameters yielding the best fits to the ambiguous-cue data alone. (B) Ambiguous-cue performance with parameters yielding the best fits to the ambiguous-cue and three-pair loop data combined. (C) Performance in the threepair loop problem with parameters yielding the best fits to the ambiguous-cue and three-pair loop data combined. (D) Predicted performance in the four-pair loop problem with parameters yielding the best fits to the ambiguouscue and three-pair loop data combined. 
PUC MODEL
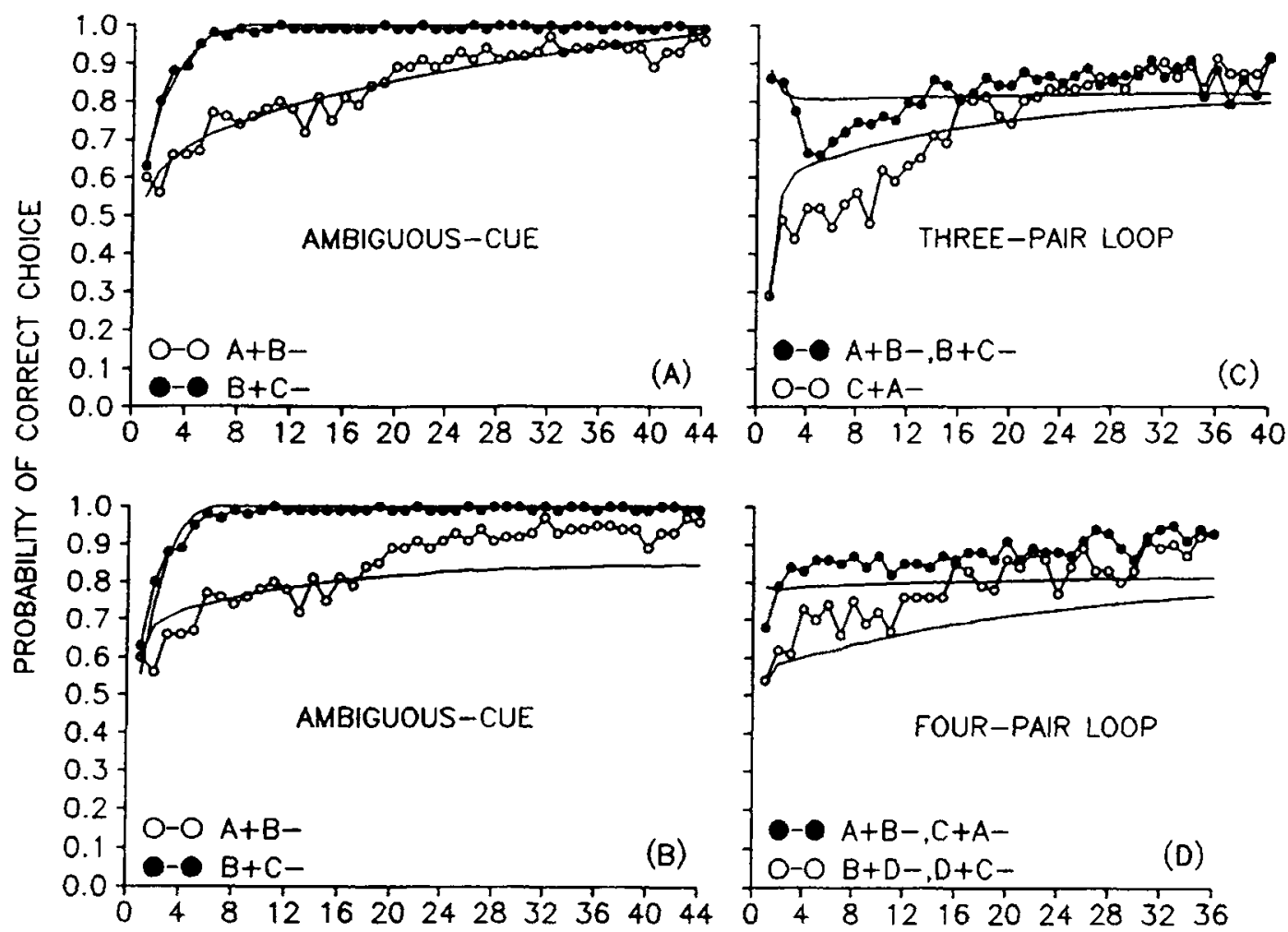

SESSIONS

Figure 6. Simulations with the PUC model (smooth curves). (A) Ambiguous-cue performance with parameters yielding the best fits to the ambiguous-cue data alone. (B) Ambiguous-cue performance with parameters yielding the best fits to the ambiguous-cue and three-pair loop data combined. (C) Performance in the three-pair loop problem with parameters yielding the best fits to the ambiguous-cue and three-pair loop data combined. (D) Predicted performance in the four-pair loop problem with parameters yielding the best fits to the ambiguous-cue and three-pair loop data combined.

tal data, in Figure 5A; for the PUC model, in Figure 6A; for the RW model, in Figure 7A; for the LS model, in Figure 8A; and for the SM model, in Figure 9A. The fitted curves are smooth because the computational method eliminates the variability among stat-animals inherent in the Monte Carlo method. The success of all five models suggests that the ambiguous-cue problem is not very constraining, although - contrary to our own expectationswe have been unable to fit the ambiguous-cue data with a continuity model that assumes no afferent interaction (i.e., relies solely on the primary components). In any event, the three-pair loop problem is more constraining. With the parameters that provide the best fits to the ambiguous-cue data alone, none of the five models yields an even remotely acceptable fit to the three-pair loop data: All but the RW model predict very strong and persistent difficulty with the new pair; the RW model predicts $\mathrm{A}+\mathrm{B}-$ much better than $\mathrm{B}+\mathrm{C}-$ performance.

Figures $5 \mathrm{~B}$ through $9 \mathrm{~B}$ are like Figures $5 \mathrm{~A}$ through $9 \mathrm{~A}$ except that the simulations are computed on the basis of the parameter values that yielded the best fits to the combined data of Stages 1 and 2 (the ambiguous-cue data and the three-pair loop data together). Here we see somewhat less agreement among the models than before (the mean of the 10 best fits for each is plotted) and poorer agreement with the data as well: There is a tendency to overestimate the initial rate of improvement in the A+Bperformance, or to underestimate its terminal level, or some of both. The PUC and LS models, which fit the $\mathrm{B}+\mathrm{C}-$ curve nicely, most closely approximate the initial rate of improvement in the $A+B-$ periormance, but most markedly underestimate its terminal level.

Figures $5 \mathrm{C}$ through $9 \mathrm{C}$ show, for each model, the mean of the 10 best fits to the three-pair loop data computed on the basis of the parameter values that yielded the best fits to the ambiguous-cue and the loop data together. To simplify the picture, the experimental results for $\mathrm{A}+\mathrm{B}-$ and $\mathrm{B}+\mathrm{C}-$ (the old pairs), which do not differ significantly from each other, are pooled, and so also are the simulated results for those pairs. Here again there is little basis for choosing among the models, all of which reflect certain global features of the data, but fail on other counts: The challenge seems to be to capture the sharp initial decline in the oldpair performance without overestimating the initial rate of 
RW MOOEL
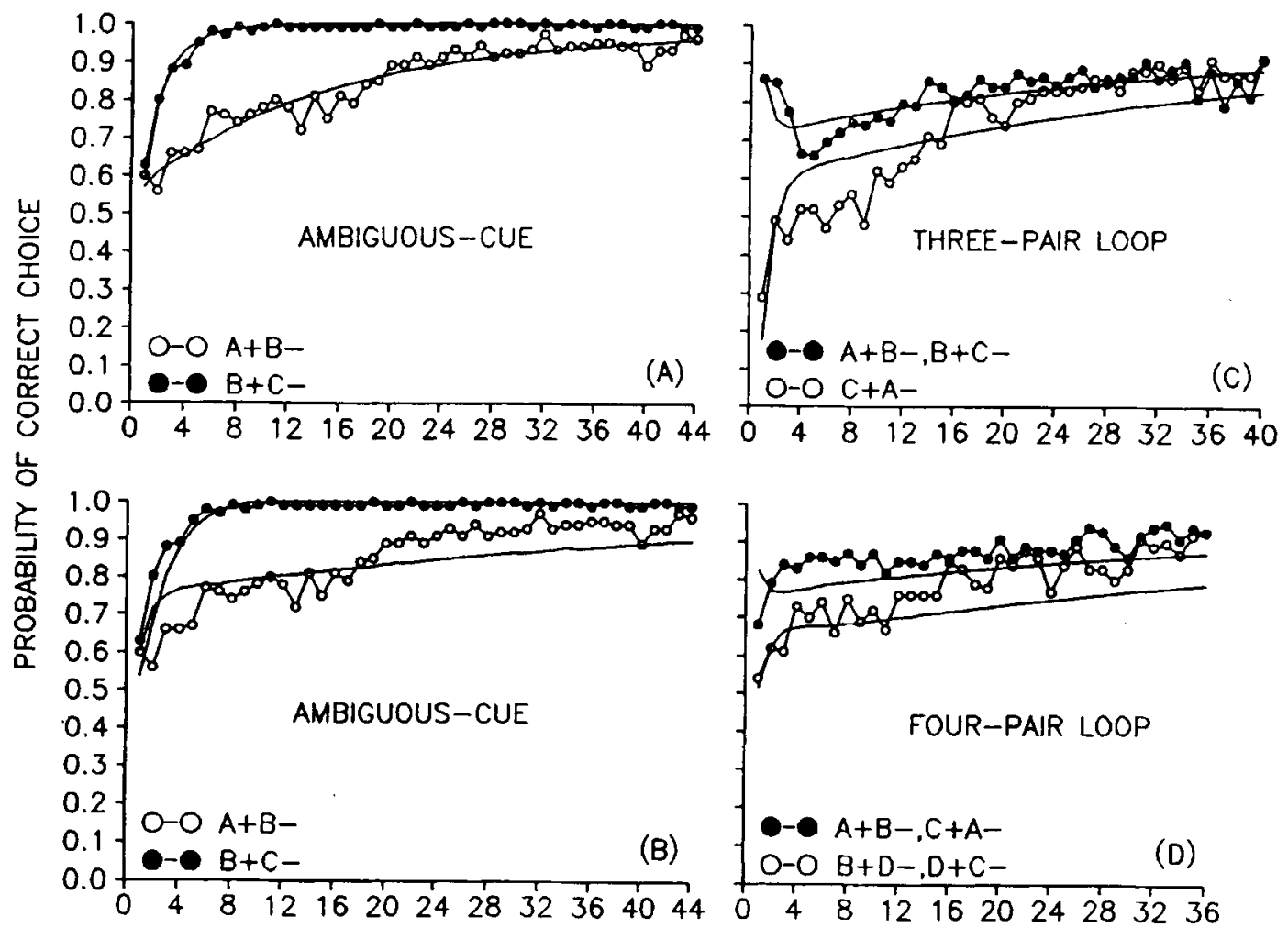

SESSIONS

Figure 7. Simulations with the RW model (smooth curves). (A) Ambiguous-cue performance with parameters yielding the best fits to the ambiguous-cue data alone. (B) Ambiguous-cue performance with parameters yielding the best fits to the ambiguous-cue and three-pair loop data combined. (C) Performance in the three-pair loop problem with parameters yielding the best fits to the ambiguous-cue and three-pair loop data combined. (D) Predicted performance in the four-pair loop problem with parameters yielding the best fits to the ambiguous-cue and three-pair loop data combined.

improvement in the new-pair $(\mathrm{C}+\mathrm{A}-)$ performance. With parameters that provide the best fits to the three-pair loop data alone, the rate of improvement in the new-pair performance is not so badly overestimated, but the fits to the ambiguouscue data are extremely inadequate: Rate of acquisition is markedly underestimated, especially for $\mathrm{A}+\mathrm{B}-$, and asymptotic levels are also underestimated, especially for $\mathrm{B}+\mathrm{C}-$.

Table 1 displays, for each model, the parameter values that yielded the 10 best fits to the ambiguous-cue and three-pair loop data together. The entries show a good deal of variability from one model to the next as well as some similarities. In the $\mathrm{HG}(\mathrm{GW})$ case, choice is governed by the matching rule ( $K=1, s=.5$ ), but the choice function of best fit in all four other cases is a considerably steeper line ( $K=1, s=.833$ ). There is some agreement, too, that the degree of interaction is rather limited. In $\mathrm{HG}(\mathrm{GW}), g$ is large; in PUC and RW, $\alpha_{U}$ is small; and in LS, where $\alpha_{U}$ does not have a fixed value, it grows slowly in the course of training and remains small (.05-.07 at the end of Stage 2). In simulating the performance of rats and rabbits in compound-component discriminations, Bellingham et al. (1985) were also required to assume low salience for compound-unique components. Nevertheless-despite their low salience - the pair-unique components play an important role in performance by virtue of the associative strength they acquire. In PUC and LS, the associative strengths of $Q, S$, and $U$ (the reinforced pair-unique components) approach 1 ; in RW, their strengths are less (about .2 at the end of Stage 2), but R, T, and W (nonreinforced pair-unique components) become inhibitory (about -.3 ). In the SM model, which provides no estimate of the degree of interaction, the pair-unique components also acquire a great deal of associative strength as the probability of attending to them increases (however slowly) from 0 at the outset of Stage 1 to about .7 at the end of Stage 2 .

As might be expected from the relatively poor fits to the data of the first and second stages, the fits of the five models to the four-pair loop data of the third stage are poor as well. Plotted in Figures 5D through 9D are the predictions (with the parameters shown in Table 1) for each of the models. Again for purposes of clarity, the experimental results for the two old pairs (here $A+B-$ and $\mathrm{C}+\mathrm{A}-$ ), which do not differ significantly from each other, are pooled; the results for the two new pairs $(B+D-$ and 
LS MODEL
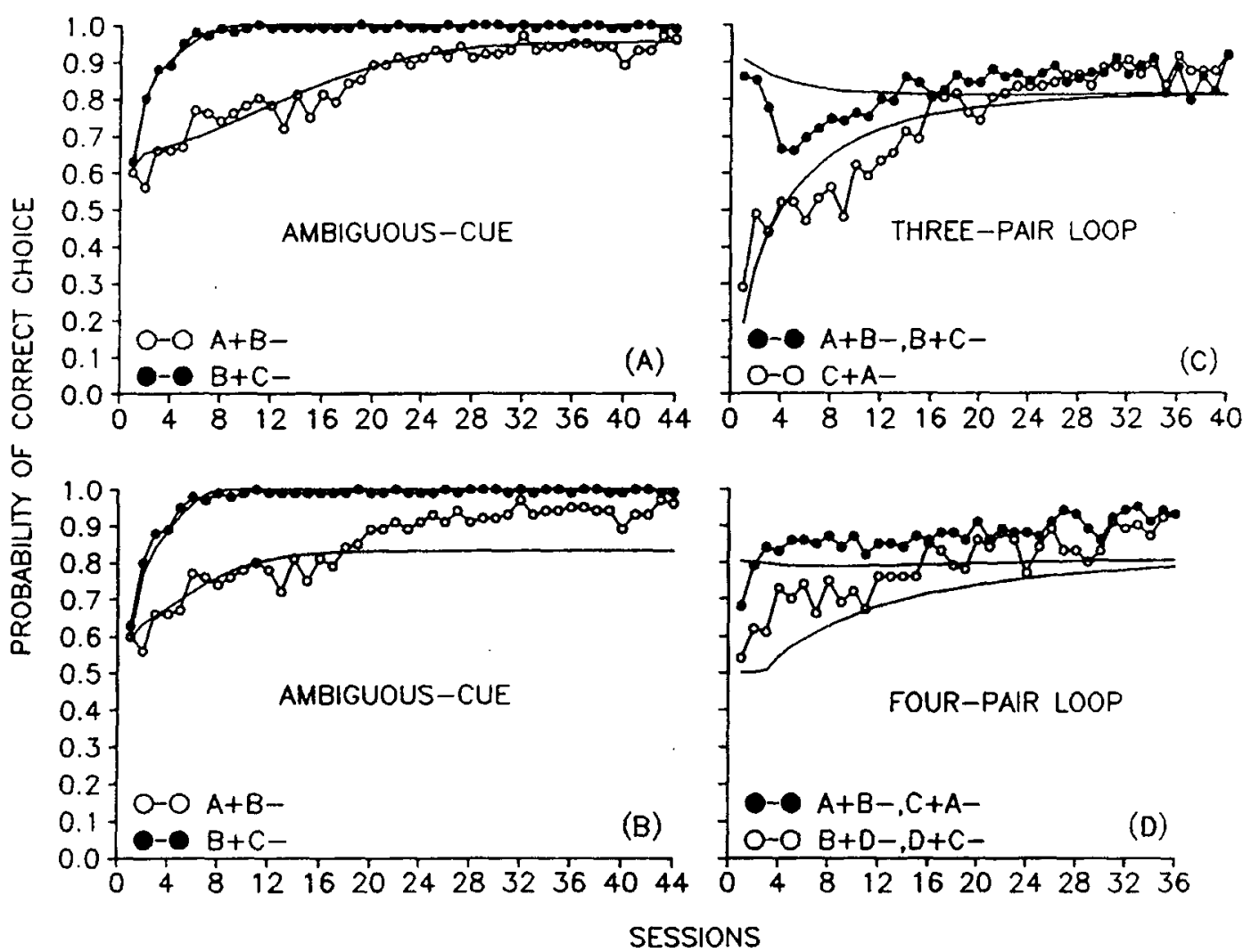

Figure 8. Simulations with the LS model (smooth curves). (A) Ambiguous-cue performance with parameters yielding the best fits to the ambiguous-cue data alone. (B) Ambiguous-cue performance with parameters yielding the best fits to the ambiguous-cue and three-pair loop data combined. (C) Performance in the three-pair loop problem with parameters yielding the best fits to the ambiguous-cue and three-pair loop data combined. (D) Predicted performance in the four-pair loop problem with parameters yielding the best fits to the ambiguous-cue and three-pair loop data combined.

$\mathrm{D}+\mathrm{C}-$ ), which do not differ significantly from each other, are pooled; and pooled also are the simulated results for the corresponding pairs. None of the models captures the initial decrement in the old-pair performance and the rapid recovery. All of the models underestimate the terminal level of the new-pair performance except SM, which predicts no difference between old-pair and newpair performance. The SM model also predicts a marked initial difference between the two new pairs $(B+D-$ per- formance better than $\mathrm{D}+\mathrm{C}-$ performance, although the trend in the data was the opposite) that gradually disappears in the course of training. Whatever the inadequacy of the other four models, that of the SM model is greatest.

\section{DISCUSSION}

Is it reasonable to expect that a sound theory will account for all of the available data as accurately as the

Table 1

Parameter Values for the Best Fits to the Ambiguous-Cue and Loop Data for Each of Five Models

\begin{tabular}{|c|c|c|c|c|c|}
\hline \multirow[b]{2}{*}{ Parameter } & \multicolumn{4}{|c|}{ Model } & $\mathrm{i}$ \\
\hline & $\mathrm{HG}(\mathrm{GW})$ & PUC & $\mathrm{RW}$ & LS & $\mathrm{SM}$ \\
\hline $\begin{array}{l}\text { Choice function } \\
U \beta(V)\end{array}$ & $\begin{array}{l}K=1, s=.5 \\
.001-.004\end{array}$ & $\begin{array}{l}K=1, s=.833 \\
.08-.1\end{array}$ & $\begin{array}{l}K=1, s=.833 \\
.02-.04\end{array}$ & $\begin{array}{l}K=1, s=.833 \\
.12-.22\end{array}$ & $\begin{array}{l}K=1, s=.833 \\
.004\end{array}$ \\
\hline$D \beta(V)$ & $.1-.16$ & .14 & .08 & $.14-.2$ & .04 \\
\hline$g$ & $.75-.85$ & & & & \\
\hline$\alpha_{\mathrm{P}}$ & & $.4-.7$ & $.65-1$ & & \\
\hline$\alpha_{U}$ & & .05 & .05 & .001 & \\
\hline$D \beta(\alpha)$ & & & & $.06-.08$ & \\
\hline$U \beta(a)$ & & & & & $.0002-.01$ \\
\hline$D \beta(a)$ & & & & & .002 \\
\hline
\end{tabular}


SM MODEL
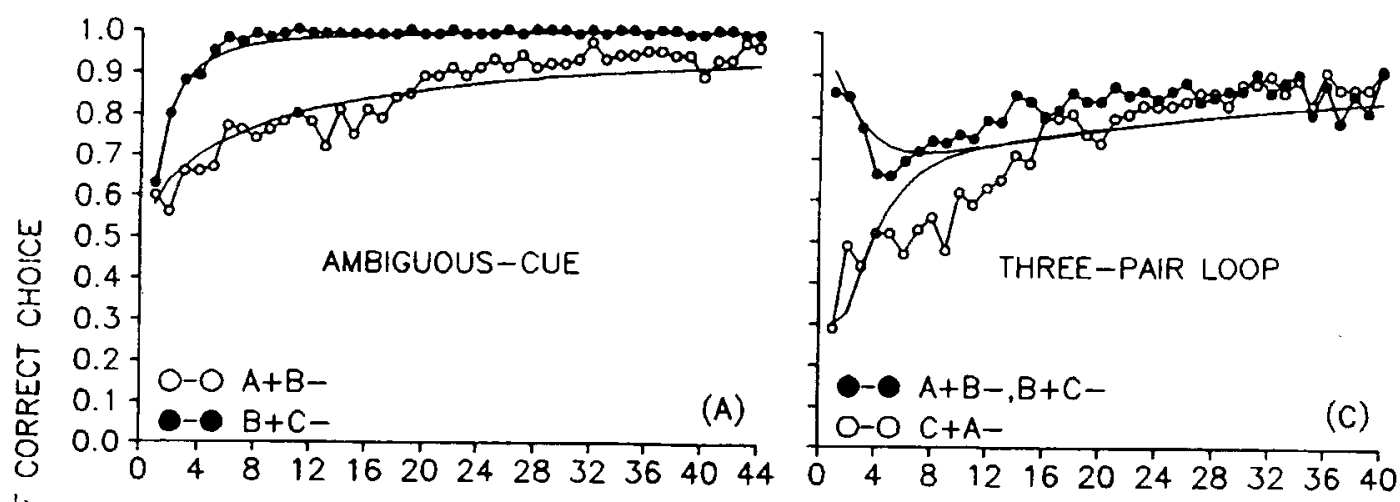

능

E
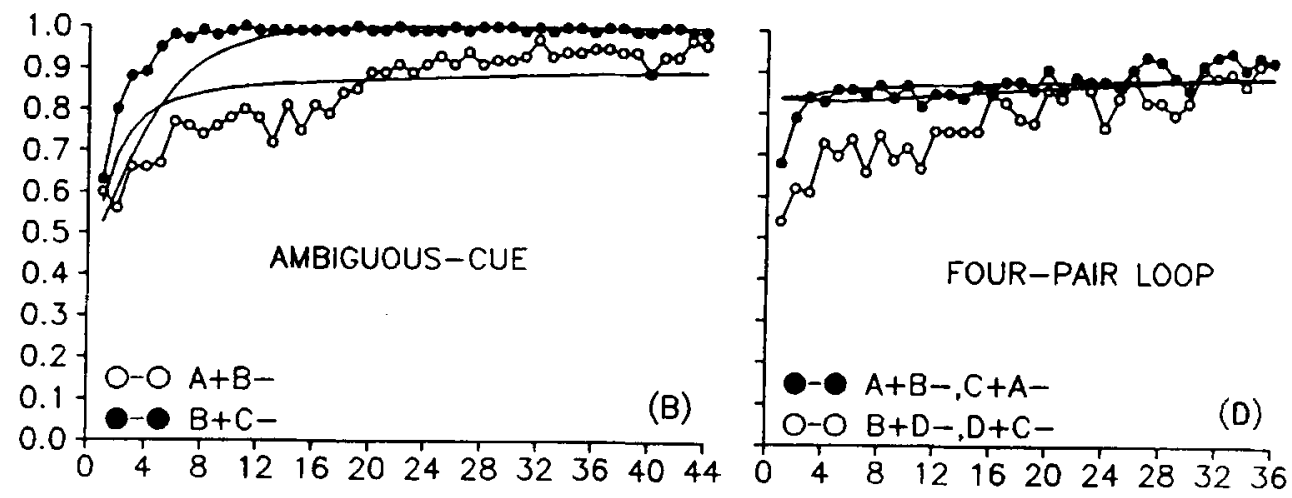

\section{SESSIONS}

Figure 9. Simulations with the SM model (smooth curves). (A) Ambiguous-cue performance with parameters yielding the best fits to the ambiguous-cue data alone. (B) Ambiguous-cue performance with parameters yielding the best fits to the ambiguous-cue and three-pair loop data combined. (C) Performance in the three-pair loop problem with parameters yielding the best fits to the ambiguous-cue and three-pair loop data combined. (D) Predicted performance in the four-pair loop problem with parameters yielding the best fits to the ambiguous-cue and three-pair loop data combined.

ambiguous-cue data alone are simulated by each of the models considered here? If so, then all of the models are suspect. The focus of difficulty is the loop problem, to the data of which none of the models yields a satisfactory fit. The SM model is distinguished by its especially poor prediction of performance in the third stage, but with that exception the models are much alike in their shortcomings despite the diversity of their key assumptions-generalization decrement in HG; pairs as configurations in GW; and pair-unique components conditioned independently in PUC, competing with the primary components for associative strength in RW, acquiring salience independently in LS, or competing with the primary components for attention in SM. From the simulation results for the $\mathrm{HG}$ and $\mathrm{GW}$ models, which seem to be saying quite different things but prove in their implementation to be identical, it is evident that linguistic diversity may mask substantive commonality. Three of the models that rely heavily on pair-unique components (PUC, RW, and LS) are not, however, functionally identical, achieving their similar yet unsatisfactory results in different ways.

It might be suggested that other variations on the same themes are worth exploring, as, for example, an LS model with shared associative strength, or an SM model with a direct (rather than an indirect) effect of attention on the growth of associative strength. A more radical departure might be prompted by Spence's advice that we should look elsewhere than to the mechanism of (unitary) compounding - to "a different set of receptor-exposure acts" (1952, p. 92), as he put it-for an explanation of transverse patterning. Although Spence theorized that there can be transverse patterning only in problems that are insoluble in terms of components alone or of compounds alone, there was little support for his view even at the time (Bitterman, 1953), and one might want to try to simulate our data with a model providing for control of performance both by pair-unique and by configurational properties, perhaps competing for attention. It is conceivable also that the correct choice function is markedly different from those we have considered here, although, as already noted, simple functions intermediate between matching and maximizing have yielded good fits to data for pigeons as well as for honeybees (Couvillon \& Bitterman, 1991, 1992). To examine any of these possibilities, or others that may suggest themselves, requires only a set of desktop computers and a degree of patience inversely related to 
their speed-patience stemming, of course, from a distrust of theories supported by merely ordinal derivations.

For the present, in any case, we are without a convincing explanation of transverse patterning. So also, by the same standard, are we without a convincing explanation of unitary compounding, since none of the proposed alternatives has undergone anything so rigorous as the testing to which we have subjected theories of transverse patterning inspired by them. Some interesting efforts to model unitary compounding have been made (Bellingham et al., 1985; Wilson \& Pearce, 1989) that have been recognized by their authors to be inadequate for the lack of performance equations required for exact predictions, but that promise if carried further to contribute to our understanding of both unitary compounding and transverse patterning.

\section{REFERENCES}

Alvarado, M. C., \& Rudy, J. W. (1992). Some properties of configural learning: An investigation of the transverse-patterning problem. Journal of Experimental Psychology: Animal Behavior Processes, 18, 145-153.

Bellingham, W. P., Gillette-Bellingham, K., \& Kehoe, E. J. (1985). Summation and configuration in patterning schedules with the rat and rabbit. Animal Learning \& Behavior, 13, 152-164.

Bitterman, M. E. (1953). Spence on the problem of patterning. $P_{s y-}$ chological Review, 60, 123-126.

BitTERman, M. E. (1984). Migration and learning in fishes. In J. D. McCleave, G. P. Arnold, J. J. Dodson, \& W. H. Neill (Eds.), Mechanisms of migration in fishes (pp. 397-420). New York: Plenum.

BOYER, W. N., \& POLIDORA, V. J. (1972). An analysis of the solution of PAN ambiguous-cue problems by rhesus monkeys. Learning \& $\mathrm{Mo}$ tivation, 3, 325-333.

BUSH, R. R., \& MostelleR, F. (1951). A mathematical model for simple learning. Psychological Review, 58, 313-323.

Couvillon, P. A., \& BitTerman, M. E. (1988). Compound-component and conditional discrimination of colors and odors by honeybees. $A n$ imal Learning \& Behavior, 16, 67-74.

Couvillon, P. A., \& BitTerman, M. E. (1989). Reciprocal overshadowing in the discrimination of color-odor compounds by honeybees: Further tests of a continuity model. Animal Learning \& Behavior, 17, 213-222.

Couvillon, P. A., \& Bitterman, M. E. (1991). How honeybees make choices. In J. L. Goodman \& R. C. Fischer (Eds.), The behaviour and physiology of bees (pp. 116-130). Wallingford, U.K.: CAB International.

Couvillon, P. A., \& Bitterman, M. E. (1992). A conventional conditioning analysis of "transitive inference" in pigeons. Journal of Experimental Psychology: Animal Behavior Processes, 18, 308-310.

Fersen, L. von, Wynne, C. D. L., Delius, J. D., \& Staddon, J. E. R. (1991). Transitive inference formation in pigeons. Journal of Experimental Psychology: Animal Behavior Processes, 17, 334-341.

Fischer, M. E., Couvillon, P. A., \& Bitterman, M. E. (1993). Choice in honeybees as a function of probability of reward. Animal Learning \& Behavior, 21, 187-195.

Gonzalez, R. C., Berger, B. D., \& Birterman, M. E. (1966). A further comparison of key-pecking with an ingestive technique for the study of discriminative learning in pigeons. American Journal of $P$ sychology, 79, 217-223.

Gulliksen, H., \& WOlfle, D. L. (1938). A theory of learning and transfer. Psychometrika, 3, 127-149.

HALL, G. (1980). An investigation of ambiguous-cue learning in pigeons. Animal Learning \& Behavior, 8, 282-286.

HeinemanN, E. G., \& Chase, S. (1970). Conditional stimulus control. Journal of Experimental Psychology, 84, 187-197.
HulL, C. L. (1943). Principles of behavior. New York: Appleton-Century. KLÜVER, H. (1933). Behavior mechanisms in monkeys. Chicago: Unjversity of Chicago Press.

LASHLEY, K. S. (1938). Conditional reactions in the rat. Journal of Psychology, 6, 311-324.

Nissen, H. W. (1950). Description of the learned response in discrimination behavior. Psychological Review, 57, 121-131.

NorTH, A. J., Maller, O., \& Hughes, C. (1958). Conditional discrimination and stimulus patterning. Journal of Comparative \& Physiological Psychology, 51, 711-715.

Pavlov, I. P. (1927). Conditioned reflexes. (G. V. Anrep, Ed. \& Trans.). London: Oxford University Press.

Pearce, J. M., \& Redhead, E. S. (1993). The influence of an irrelevant stimulus on two discriminations. Journal of Experimental Psychology: Animal Behavior Processes, 19, 180-190.

Pearce, J. M., \& Wilson, P. N. (1990). Configural associations in discrimination learning. Journal of Experimental Psychology: Animal Behavior Processes, 16, 250-261.

ResCORLA, R. A. (1972). "Configural" conditioning in discrete-trial bar pressing. Journal of Comparative \& Physiological Psychology, 79, 307-317.

Rescorla, R. A., Grau, J. W., \& Durlach, P. J. (1985). Analysis of the unique cue in configural discriminations. Journal of Experimental Psychology: Animal Behavior Processes, 11, 356-366.

Rescorla, R. A., \& WAGNER, A. R. (1972). A theory of classical conditioning: Variation in the effectiveness of reinforcement and nonreinforcement. In A. H. Black \& W. F. Prokasy (Eds.), Classical conditioning II: Current research and theory (pp. 64-99). New York: Appleton-Century-Crofts.

SaAVEDRA, M. A. (1975). Pavlovian compound conditioning in the rabbit. Learning \& Motivation, 6, 314-326.

SPENCE, K. W. (1952). The nature of response in discrimination learning. Psychological Review, 59, 89-93.

Stearns, E. M., \& Bitterman, M. E. (1965). A comparison of keypecking with an ingestive technique for the study of discriminative learning in pigeons. American Journal of Psychology, 78, 48-56.

Sutherland, N. S., \& MaCkINTOSH, N. J. (1971). Mechanisms of animal discrimination learning. New York: Academic Press.

TEas, D. C., \& Bitrerman, M. E. (1952). Perceptual organization in the rat. Psychological Review, 59, 130-140.

THOMPSON, R. (1953). Approach-avoidance in an ambivalent object discrimination problem. Journal of Experimental Psychology, 45, 341-344.

Turbeville, J. R., Calvin, A. D., \& BitTerman, M. E. (1952). Relational and configurational learning in the rat. American Journal of Psychology, 65, 424-433.

WhitLoW, J. W., JR., \& WAGNER, A. R. (1972). Negative patterning in classical conditioning: Summation of response tendencies in isolable and configural components. Psychonomic Science, 27, 299-301.

Wilson, P. N., \& PearCe, J. M. (1989). A role for stimulus generalization in conditional discrimination learning. Quarterly Journal of Experimental Psychology, 41B, 243-273.

Wodinsky, J., \& BitTerMAN, M. E. (1953). The solution of oddity problems by the rat. American Journal of Psychology, 66, 137-140.

Woodbury, C. B. (1943). The learning of stimulus patterns by dogs. Journal of Comparative Psychology, 35, 29-40.

WYNNE, C. D. L. (1995). Reinforcement accounts for transitive inference. Animal Learning \& Behavior, 23, 207-217.

ZEILER, M. D., \& PAUL, B. J. (1965). Intra-pair similarity as a determinant of component and configuration discrimination. American Journal of Psychology, 78, 476-480.

Zhuikov, A. Y., Couvillon, P. A., \& Bitterman, M. E. (1994). Quantitative two-process analysis of avoidance conditioning in goldfish. Journal of Experimental Psychology, 20, 32-43.

(Manuscript received August 30, 1995; revision accepted for publication December 8,1995 .) 